\title{
Hybrid mean value for a generalization of a problem of D. H. Lehmer
}

\author{
by
}

\author{
Huaning Liu and Wenpeng Zhang (Xi'an)
}

1. Introduction. Let $q>2$ be an odd number and $c$ be any integer with $(c, q)=1$. For each integer $a$ with $1 \leq a \leq q$ and $(a, q)=1$, we know that there exists one and only one $b$ with $1 \leq b \leq q$ such that $a b \equiv c \bmod q$. Let $N(q, c)$ be the number of cases in which $a$ and $b$ are of opposite parity, that is,

$$
N(q, c)=\sum_{\substack{a=1 \\ a b \equiv \operatorname{cmod} q \\ 2 \nmid a+b}}^{q} \sum_{\substack{b=1 \\ \text { a }}}^{\prime} 1,
$$

where $\sum_{a=1}^{\prime q}$ denotes summation over all $a$ such that $(a, q)=1$. For $c=1$ and $q=p$ an odd prime, D. H. Lehmer [3] asked to find $N(p, 1)$ or at least to say something nontrivial about it. In [6] and [7], the second author proved that

$$
N(q, 1)=\frac{1}{2} \phi(q)+O\left(q^{1 / 2} d^{2}(q) \ln ^{2} q\right),
$$

where $\phi(q)$ is the Euler function, and $d(q)$ is the divisor function. For any nonnegative integer $n$, let

$$
N(q, 1, n)=\sum_{\substack{a=1 \\ a b \equiv 1 \bmod q \\ 2 \nmid a+b}}^{q} \sum_{\substack{b=1 \\ q}}^{\prime}(a-b)^{2 n} .
$$

2000 Mathematics Subject Classification: Primary 11L05.

Key words and phrases: Lehmer problem, Cochrane sums.

Research partially supported by the National Natural Science Foundation of China under Grants No. 60472068 and No. 10671155; Natural Science Foundation of Shaanxi province of China under Grant No. 2006A04; and the Natural Science Foundation of the Education Department of Shaanxi province of China under Grant No. 06JK168. 
The second author [8] proved the following asymptotic formula:

$$
N(q, 1, n)=\frac{1}{(2 n+1)(2 n+2)} \phi(q) q^{2 n}+O\left(4^{n} q^{2 n+1 / 2} d^{2}(q) \ln ^{2} q\right) .
$$

For any fixed positive integer $c$ with $(c, q)=1$, let

$$
E(q, c)=N(q, c)-\frac{1}{2} \phi(q) .
$$

The second author [11] showed that for any odd prime $p$,

$$
\sum_{c=1}^{p-1}|E(p, c)|^{2}=\frac{3}{4} p^{2}+O\left(p \exp \left(\frac{3 \ln p}{\ln \ln p}\right)\right) .
$$

This proves the error term in (1) is the best possible. In [12], he found a close relation between the error terms $E(q, c)$ and the classical Kloosterman sums

$$
K(m, n ; q)=\sum_{b=1}^{q} e\left(\frac{m b+n \bar{b}}{q}\right)
$$

where $e(y)=e^{2 \pi i y}$, and obtained the following hybrid mean value formula:

$$
\sum_{c=1}^{q} E(q, c) K(\overline{4} c, 1 ; q)=\frac{4}{\pi^{2}} q \phi(q) \prod_{p \| q}\left(1-\frac{1}{p(p-1)}\right)+O\left(q^{3 / 2+\varepsilon}\right),
$$

where $4 \overline{4} \equiv 1 \bmod q, \varepsilon$ is any fixed positive number, and $\prod_{p \|_{q}}$ denotes the product over all prime divisors of $q$ with $p \mid q$ and $p^{2} \nmid q$.

It is natural and interesting to study the hybrid mean value between the error terms in this problem of D. H. Lehmer and other arithmetic functions. Professor Todd Cochrane introduced a sum analogous to the Dedekind sum as follows:

$$
C(h, q)=\sum_{a=1}^{q}\left(\left(\frac{\bar{a}}{q}\right)\right)\left(\left(\frac{a h}{q}\right)\right),
$$

where $\bar{a}$ is defined by the equation $a \bar{a} \equiv 1 \bmod q$ and

$$
((x))= \begin{cases}x-[x]-1 / 2 & \text { if } x \text { is not an integer, } \\ 0 & \text { if } x \text { is an integer. }\end{cases}
$$

Then he suggested studying the arithmetical properties and mean value distribution properties of $C(h, q)$. The second author and Y. Yi [14] proved that $|C(h, k)| \ll \sqrt{k} d(k) \ln ^{2} k$, and obtained some interesting hybrid mean value formulae between Cochrane sums and Kloosterman sums (see [9], [10] and [13]). 
Now we consider a generalization of this problem of D. H. Lehmer. For any integer $k \geq 1$, let

$$
\begin{aligned}
& M(p, k, c)=\sum_{\substack{a_{1}=1 \\
a_{1} \cdots a_{k} b \equiv c \bmod p \\
2 \nmid a_{1}+\cdots+a_{k}+b}}^{p-1} \cdots \sum_{\substack{a_{k}=1 \\
b=1}}^{p-1} \sum^{p-1}\left(a_{1}+\cdots+a_{k}-b\right)^{2}, \\
& E(p, k, c)=M(p, k, c)-\frac{\left(3 k^{2}-5 k+4\right) p-2 k-2}{24} p(p-1)^{k} .
\end{aligned}
$$

In this paper, we use the Fourier expansion for character sums and the mean value theorems for Dirichlet $L$-functions to study the hybrid mean value of $E(p, k, c)$ and the $k$-dimensional Cochrane sums

$$
C(c, k, p)=\sum_{a_{1}=1}^{p-1} \cdots \sum_{a_{k}=1}^{p-1}\left(\left(\frac{\bar{a}_{1}}{p}\right)\right) \cdots\left(\left(\frac{\bar{a}_{k}}{p}\right)\right)\left(\left(\frac{a_{1} \cdots a_{k} c}{p}\right)\right),
$$

and give two interesting asymptotic formulae. Namely, we prove the following:

THEOREM 1. For any prime $p \geq 3$, we have the asymptotic formula

$$
\sum_{c=1}^{p-1} E(p, 1, c) C(c, 1, p)=-\frac{5}{144} p^{4}+O\left(p^{7 / 2+\varepsilon}\right),
$$

where $\varepsilon$ is any fixed positive number.

TheOREM 2. For any prime $p \geq 3$ and integer $k \geq 2$, we have

$$
\begin{aligned}
& \sum_{c=1}^{p-1} E(p, k, c) C(c, k, p) \\
& =-\frac{2^{k-2}\left(k^{2}-k+2\right) \zeta^{2 k+1}(2) p^{k+3}}{\pi^{2 k+2}}\left[\sum_{i=0}^{k+1} C_{k+1}^{i}(-2)^{i} C_{k}(i)\right] \\
& \quad \times \prod_{p_{1} \nmid 2 p}\left(1-\frac{1-C_{2 k}^{k}}{p_{1}^{2}}\right) \\
& \quad+\frac{2^{k-1}(k+1) p^{k+3}}{\pi^{2 k+4}}\left[\sum_{i=0}^{k} C_{k}^{i}(-2)^{i} \frac{D_{k}(2, i)}{2^{3 i+1}}+\sum_{j=0}^{k} C_{k}^{j}(-2)^{j+3} \frac{D_{k}(2, j+1)}{2^{3 j+4}}\right] \\
& \quad \times \prod_{p_{1} \nmid 2 p} D_{k}\left(p_{1}, 0\right)+O\left(p^{k+5 / 2+\varepsilon}\right),
\end{aligned}
$$

where $\zeta(s)$ is the Riemann zeta-function, $\prod_{p_{1} \nmid 2 p}$ denotes the product over 
all different primes $p_{1}$ with $p_{1} \nmid 2 p$,

$$
\begin{aligned}
C_{k}(i) & =\frac{1}{2^{i+1}}\left[\left(1-\frac{1}{2^{2}}\right) C_{k+i}^{k}+\frac{C_{2 k}^{k}}{2^{2}}\right], \\
D_{k}(p, i) & =\sum_{n=0}^{\infty} \frac{C_{k+n}^{k}}{p^{4 n}} \sum_{j=0}^{i+n} p^{2 j} C_{k+j-1}^{k-1},
\end{aligned}
$$

and $C_{m}^{n}=m ! / n !(m-n) !$.

2. Some lemmas. To prove the theorems, we need the following lemmas.

LEMMA 1. Let $\chi$ be a primitive character modulo $q$ with $\chi(-1)=-1$, and $q \geq 3$ an odd number. Then

$$
\begin{aligned}
\sum_{a=1}^{q} a \chi(a) & =\frac{q i}{\pi} \tau(\chi) L(1, \bar{\chi}) \\
\sum_{a=1}^{q}(-1)^{a} \chi(a)= & \frac{2(1-2 \chi(2)) i}{\pi} \tau(\chi) L(1, \bar{\chi}), \\
\sum_{a=1}^{q}(-1)^{a} a \chi(a)= & \frac{q(1-2 \chi(2)) i}{\pi} \tau(\chi) L(1, \bar{\chi}), \\
\sum_{a=1}^{q}(-1)^{a} a^{2} \chi(a)= & \frac{q^{2}(1-2 \chi(2)) i}{\pi} \tau(\chi) L(1, \bar{\chi}) \\
& +\frac{q^{2}(8 \chi(2)-1) i}{\pi^{3}} \tau(\chi) L(3, \bar{\chi})+O\left(q^{2}\right),
\end{aligned}
$$

where $L(s, \chi)$ is the Dirichlet L-function corresponding to $\chi, \tau(\chi)=$ $\sum_{a=1}^{q} \chi(a) e(a / q)$ is the Gauss sum, and $|\tau(\chi)|=\sqrt{q}$.

Proof. For any odd character $\chi$ modulo $q$, it is easy to show that

$$
\begin{aligned}
\sum_{a=1}^{q}(-1)^{a} a \chi(a) & =\frac{q}{2} \sum_{a=1}^{q}(-1)^{a} \chi(a), \\
\sum_{b=1}^{q}(-1)^{b} \chi(b) & =2 \chi(2) \sum_{b=1}^{(q-1) / 2} \chi(b) .
\end{aligned}
$$

From [2] we have the identity

$$
(1-2 \chi(2)) \sum_{c=1}^{q} c \chi(c)=\chi(2) q \sum_{c=1}^{(q-1) / 2} \chi(c) .
$$


On the other hand, from Theorems 12.11 and 12.20 of [1] we know that if $\chi$ is a primitive character modulo $q$ with $\chi(-1)=-1$, then

$$
\frac{1}{q} \sum_{b=1}^{q} b \chi(b)=\frac{i}{\pi} \tau(\chi) L(1, \bar{\chi}) .
$$

This yields formulae (2)-(4).

For any odd primitive character $\chi$ modulo $q$, we also have

$$
\begin{aligned}
\sum_{a=1}^{q}(-1)^{a} a^{2} \chi(a) & =\sum_{\substack{a=1 \\
2 \mid a}}^{q} a^{2} \chi(a)-\sum_{\substack{a=1 \\
2 \nmid a}}^{q} a^{2} \chi(a) \\
& =\sum_{\substack{a=1 \\
2 \mid a}}^{q} a^{2} \chi(a)-\sum_{\substack{a=1 \\
2 \mid a}}^{q}(q-a)^{2} \chi(q-a) \\
& =2 \sum_{a=1} a^{2} \chi(a)-2 q \sum_{a=1}^{q} a \chi(a)+q^{2} \sum_{a=1}^{q} \chi(a) . \\
2 \mid a &
\end{aligned}
$$

Noting that

$$
\sum_{a=1}^{q} a \chi(a)=\sum_{\substack{a=1 \\ 2 \mid a}}^{q} a \chi(a)+\sum_{\substack{a=1 \\ 2 \mid a}}^{q}(q-a) \chi(q-a)=2 \sum_{\substack{a=1 \\ 2 \mid a}}^{q} a \chi(a)-q \sum_{\substack{a=1 \\ 2 \mid a}}^{q} \chi(a)
$$

from (8) and (9) we get

$$
\begin{aligned}
\sum_{a=1}^{q}(-1)^{a} a^{2} \chi(a) & =2 \sum_{\substack{a=1 \\
2 \mid a}}^{q} a^{2} \chi(a)-q \sum_{a=1}^{q} a \chi(a) \\
& =8 \chi(2) \sum_{a=1}^{q / 2} a^{2} \chi(a)-\frac{q^{2} i}{\pi} \tau(\chi) L(1, \bar{\chi}) .
\end{aligned}
$$

Noticing the Fourier expansion for character sums which was first given by Pólya [5]:

$$
\sum_{0<n \leq q y} \chi(n)= \begin{cases}\frac{\tau(\chi)}{\pi} \sum_{n=1}^{\infty} \frac{\bar{\chi}(n) \sin (2 \pi n y)}{n}+O(1) & \text { if } \chi(-1)=1, \\ \frac{\tau(\chi) L(1, \bar{\chi})}{\pi i}-\frac{\tau(\chi)}{\pi i} \sum_{n=1}^{\infty} \frac{\bar{\chi}(n) \cos (2 \pi n y)}{n}+ & O(1) \\ & \text { if } \chi(-1)=-1,\end{cases}
$$

where $\chi$ is a primitive character modulo $q$, and $y>0$ is a real number, by 
Abel's identity we have

$$
\begin{aligned}
& \sum_{n=1}^{q / 2} n^{2} \chi(n)=\frac{q^{2}}{4} \sum_{0<n \leq q / 2} \chi(n)-2 \int_{0}^{q / 2} u \sum_{0<n \leq u} \chi(n) d u \\
& =\frac{q^{2}(\bar{\chi}(2)-2) i}{4 \pi} \tau(\chi) L(1, \bar{\chi})-2 \int_{0}^{q / 2} u \sum_{0<n \leq u} \chi(n) d u \\
& =\frac{q^{2}(\bar{\chi}(2)-2) i}{4 \pi} \tau(\chi) L(1, \bar{\chi})-2 q^{2} \int_{0}^{1 / 2} s \sum_{0<n \leq q s} \chi(n) d s \\
& =\frac{q^{2}(\bar{\chi}(2)-2) i}{4 \pi} \tau(\chi) L(1, \bar{\chi}) \\
& \quad-2 q^{2} s\left[\frac{\tau(\chi) L(1, \bar{\chi})}{\pi i}-\frac{\tau(\chi)}{\pi i} \sum_{n=1}^{\infty} \frac{\bar{\chi}(n) \cos (2 \pi n s)}{n}+O(1)\right] d s \\
& =\frac{q^{2}(\bar{\chi}(2)-1) i}{4 \pi} \tau(\chi) L(1, \bar{\chi})+2 q^{2} \frac{\tau(\chi)}{\pi i} \sum_{n=1}^{\infty} \frac{\bar{\chi}(n)}{n} \int_{0}^{1 / 2} s \cos (2 \pi n s) d s+O\left(q^{2}\right) \\
& =\frac{q^{2}(\bar{\chi}(2)-1) i}{4 \pi} \tau(\chi) L(1, \bar{\chi})+\frac{q^{2} \tau(\chi)}{2 \pi^{3} i} \sum_{n=1}^{\infty} \frac{\bar{\chi}(n)(\cos (\pi n)-1)}{n^{3}}+O\left(q^{2}\right) \\
& =\frac{q^{2}(\bar{\chi}(2)-1) i}{4 \pi} \tau(\chi) L(1, \bar{\chi})-\frac{q^{2} \tau(\chi)}{\pi^{3} i} \sum_{n=1}^{\infty} \frac{\bar{\chi}(n)}{n^{3}}+O\left(q^{2}\right) \\
& =\frac{q^{2}(\bar{\chi}(2)-1) i}{4 \pi} \tau(\chi) L(1, \bar{\chi})+\frac{q^{2}(\bar{\chi}(2)-8)}{8 \pi^{3} i} \tau(\chi) L(3, \bar{\chi})+O\left(q^{2}\right), \\
& =0
\end{aligned}
$$

so from (10) we obtain (5).

Lemma 2. Let $p$ be an odd prime. Then

$$
\begin{aligned}
E(p, 1, c)= & \frac{1}{p-1} \sum_{\substack{\chi(-1)=1 \\
\chi \neq \chi_{0}}} \bar{\chi}(c)\left(\sum_{a=1}^{p}(-1)^{a} a \chi(a)\right)^{2} \\
& -\frac{1}{p-1} \sum_{\chi(-1)=-1} \bar{\chi}(c)\left[\left(\sum_{a=1}^{p} a \chi(a)\right)^{2}\right. \\
& \left.+\left(\sum_{a=1}^{p}(-1)^{a} a^{2} \chi(a)\right)\left(\sum_{b=1}^{p}(-1)^{b} \chi(b)\right)-\left(\sum_{a=1}^{p}(-1)^{a} a \chi(a)\right)^{2}\right] \\
& +O(p),
\end{aligned}
$$


where $\sum_{\chi(-1)=1, \chi \neq \chi_{0}}$ denotes summation over all nonprincipal even characters modulo $p$, and $\sum_{\chi(-1)=-1}$ denotes the summation over all odd characters modulo $p$.

Proof. From the definition of $M(p, 1, c)$ we get

$$
\begin{aligned}
M(p, 1, c) & =\sum_{\substack{a=1 \\
a b \equiv c \bmod p \\
2 \nmid a+b}}^{p-1} \sum_{b=1}^{p-1}(a-b)^{2}=\frac{1}{2} \sum_{\substack{a=1 \\
a b \equiv c \bmod p}}^{p-1} \sum_{b=1}^{p-1}\left[1-(-1)^{a+b}\right](a-b)^{2} \\
& =\frac{1}{2} \sum_{\substack{a=1 \\
a b \equiv c \bmod p}}^{p-1} \sum_{b=1}^{p-1}(a-b)^{2}-\frac{1}{2} \sum_{\substack{a=1 \\
a b \equiv c \bmod p}}^{p-1} \sum_{b=1}^{p-1}(-1)^{a+b}(a-b)^{2} .
\end{aligned}
$$

By the orthogonality relation for character sums modulo $p$ we easily deduce

$$
\begin{aligned}
M(p, 1, c)= & \frac{p-2}{12} p(p-1)-\frac{1}{p-1} \sum_{\chi \neq \chi_{0}} \bar{\chi}(c)\left(\sum_{a=1}^{p} a \chi(a)\right)^{2}+\frac{p-1}{4} \\
& -\frac{1}{p-1} \sum_{\chi \neq \chi_{0}} \bar{\chi}(c) \\
& \times\left[\left(\sum_{a=1}^{p}(-1)^{a} a^{2} \chi(a)\right)\left(\sum_{b=1}^{p}(-1)^{b} \chi(b)\right)-\left(\sum_{a=1}^{p}(-1)^{a} a \chi(a)\right)^{2}\right] .
\end{aligned}
$$

Note that

$$
\sum_{a=1}^{p}(-1)^{b} \chi(b)=0 \quad \text { if } \chi(-1)=1
$$

and

$$
\sum_{a=1}^{p} a \chi(a)=0 \quad \text { if } \chi(-1)=1 \text { and } \chi \neq \chi_{0} .
$$

This yields the conclusion of Lemma 2 .

LEMmA 3. Let $p$ be an odd prime and $k \geq 2$ be an integer. Then $E(p, k, c)$

$$
\begin{aligned}
= & \frac{-1}{2(p-1)} \sum_{\chi(-1)=-1} \bar{\chi}(c)\left[(k+1)\left(\sum_{a=1}^{p}(-1)^{a} \chi(a)\right)^{k}\left(\sum_{b=1}^{p}(-1)^{b} b^{2} \chi(b)\right)\right. \\
& \left.+k(k-3)\left(\sum_{a=1}^{p}(-1)^{a} a \chi(a)\right)^{2}\left(\sum_{b=1}^{p}(-1)^{b} \chi(b)\right)^{k-1}\right] .
\end{aligned}
$$


Proof. From the definition of $M(p, k, c)$ we get

$$
\begin{aligned}
M(p, k, c) & =\sum_{\substack{a_{1}=1 \\
a_{1} \cdots a_{k} b \equiv c \bmod p \\
2 \nmid a_{1}+\cdots+a_{k}+b}}^{p-1} \cdots \sum_{\substack{a_{k}=1 \\
b-1}}^{p-1}\left(a_{1}+\cdots+a_{k}-b\right)^{2} \\
& =\frac{1}{2} \sum_{\substack{a_{1}=1 \\
a_{1} \cdots a_{k} b \equiv c \bmod p}}^{p-1} \cdots \sum_{\substack{a_{k}=1 \\
b=1}}^{p-1}\left[1-(-1)^{a_{1}+\cdots+a_{k}+b}\right]\left(a_{1}+\cdots+a_{k}-b\right)^{2} \\
& =: P(p, k, c)+E(p, k, c) .
\end{aligned}
$$

By the orthogonality relation for character sums modulo $p$ we deduce

$$
\begin{aligned}
& P(p, k, c)=\frac{1}{2} \sum_{\substack{a_{1}=1 \\
a_{1} \cdots a_{k} b \equiv c \bmod p}}^{p-1} \cdots \sum_{\substack{a_{k}=1 \\
b=1}}^{p-1}\left(a_{1}+\cdots+a_{k}-b\right)^{2} \\
& =\frac{1}{2(p-1)} \sum_{\chi \bmod p} \bar{\chi}(c) \sum_{a_{1}=1}^{p-1} \cdots \sum_{a_{k}=1}^{p-1} \sum_{b=1}^{p-1} \chi\left(a_{1}\right) \cdots \chi\left(a_{k}\right) \chi(b)\left(a_{1}+\cdots+a_{k}-b\right)^{2} \\
& =\frac{1}{2(p-1)} \sum_{a_{1}=1}^{p-1} \cdots \sum_{a_{k}=1}^{p-1} \sum_{b=1}^{p-1}\left(a_{1}+\cdots+a_{k}-b\right)^{2} \\
& =\frac{k+1}{2(p-1)} \sum_{a_{1}=1}^{p-1} \cdots \sum_{a_{k}=1}^{p-1} \sum_{b=1}^{p-1} b^{2}+\frac{k(k-3)}{2(p-1)} \sum_{a_{1}=1}^{p-1} \cdots \sum_{a_{k}=1}^{p-1} \sum_{b=1}^{p-1} a_{1} b \\
& =\frac{k+1}{12} p(2 p-1)(p-1)^{k}+\frac{k(k-3)}{8} p^{2}(p-1)^{k} \\
& =\frac{\left(3 k^{2}-5 k+4\right) p-2 k-2}{24} p(p-1)^{k} .
\end{aligned}
$$

On the other hand, from (11) we have

$$
\begin{aligned}
E(p, k, c)= & -\frac{1}{2} \sum_{a_{1}=1}^{p-1} \cdots \sum_{\substack{a_{k}=1 \\
a_{1} \cdots a_{k} b \equiv c \bmod p}}^{p-1} \sum_{b=1}^{p-1}(-1)^{a_{1}+\cdots+a_{k}+b}\left(a_{1}+\cdots+a_{k}-b\right)^{2} \\
= & -\frac{1}{2(p-1)} \sum_{\chi \bmod p} \bar{\chi}(c) \sum_{a_{1}=1}^{p-1} \cdots \sum_{a_{k}=1}^{p-1} \sum_{b=1}^{p-1}(-1)^{a_{1}+\cdots+a_{k}+b} \\
& \times \chi\left(a_{1}\right) \cdots \chi\left(a_{k}\right) \chi(b)\left(a_{1}+\cdots+a_{k}-b\right)^{2}
\end{aligned}
$$




$$
\begin{aligned}
& =\frac{-1}{2(p-1)} \sum_{\chi(-1)=-1} \bar{\chi}(c)\left[(k+1)\left(\sum_{a=1}^{p}(-1)^{a} \chi(a)\right)^{k}\left(\sum_{b=1}^{p}(-1)^{b} b^{2} \chi(b)\right)\right. \\
& \left.\quad+k(k-3)\left(\sum_{a=1}^{p}(-1)^{a} a \chi(a)\right)^{2}\left(\sum_{b=1}^{p}(-1)^{b} \chi(b)\right)^{k-1}\right] .
\end{aligned}
$$

This proves Lemma 3 .

LEMMA 4. Let $p$ be an odd prime and $h$ be an integer with $(h, p)=1$. Then

$$
C(h, k, p)=\frac{i^{k+1}}{\pi^{k+1}(p-1)} \sum_{\chi(-1)=-1} \bar{\chi}(h) \tau^{k+1}(\chi) L^{k+1}(1, \bar{\chi}) .
$$

Proof. From the orthogonality relation for characters modulo $p$ we have

$$
\begin{aligned}
& \text { 13) } \quad C(h, k, p)=\sum_{a_{1}=1}^{p-1} \cdots \sum_{a_{k}=1}^{p-1}\left(\left(\frac{\bar{a}_{1}}{p}\right)\right) \cdots\left(\left(\frac{\bar{a}_{k}}{p}\right)\right)\left(\left(\frac{a_{1} \cdots a_{k} h}{p}\right)\right) \\
& =\frac{1}{p-1} \sum_{\chi \bmod p}\left\{\sum_{a_{1}=1}^{p-1} \chi\left(a_{1}\right)\left(\left(\frac{a_{1}}{p}\right)\right)\right\} \cdots\left\{\sum_{a_{k}=1}^{p-1} \chi\left(a_{k}\right)\left(\left(\frac{a_{k}}{p}\right)\right)\right\}
\end{aligned}
$$$$
\times\left\{\sum_{a_{k+1}=1}^{p-1} \chi\left(a_{k+1}\right)\left(\left(\frac{a_{k+1} h}{p}\right)\right)\right\}
$$$$
=\frac{1}{p-1} \sum_{\chi \bmod p}\left\{\sum_{a=1}^{p-1} \chi(a)\left(\frac{a}{p}-\frac{1}{2}\right)\right\}^{k}\left\{\bar{\chi}(h) \sum_{a_{k+1}=1}^{p-1} \chi\left(a_{k+1} h\right)\left(\left(\frac{a_{k+1} h}{p}\right)\right)\right\}
$$$$
=\frac{1}{p-1} \sum_{\chi \bmod p} \bar{\chi}(h)\left\{\sum_{a=1}^{p-1} \chi(a)\left(\frac{a}{p}-\frac{1}{2}\right)\right\}^{k+1} \text {. }
$$

For $\chi(-1)=1$, it is easy to prove that

$$
\sum_{a=1}^{p-1} \chi(a)\left(\frac{a}{p}-\frac{1}{2}\right)=0
$$

If $\chi(-1)=-1$, then

$$
\sum_{a=1}^{p-1} \chi(a)\left(\frac{a}{p}-\frac{1}{2}\right)=\frac{1}{p} \sum_{a=1}^{p-1} a \chi(a) .
$$

Combining (8), (13), (14) and (15) we immediately obtain the conclusion of Lemma 4. 
Lemma 5. Let $q, k, i$ be integers with $q \geq 3, k \geq 1$ and $i \geq 0$. Then we have the asymptotic formulae

$$
\begin{aligned}
& \sum_{\substack{\chi \bmod q \\
\chi(-1)=-1}} \chi\left(2^{i}\right)|L(1, \bar{\chi})|^{2 k+2} \\
& \quad=C_{k}(i) \zeta^{2 k+1}(2) \phi(q) \prod_{p \mid q}\left(1-\frac{1}{p^{2}}\right)^{2 k+1} \prod_{p \nmid 2 q}\left(1-\frac{1-C_{2 k}^{k}}{p^{2}}\right)+O\left(q^{\varepsilon}\right)
\end{aligned}
$$

and

$$
\sum_{\substack{\chi \bmod q \\ \chi(-1)=-1}} \chi\left(2^{i}\right) L^{k+1}(1, \chi) L^{k}(1, \bar{\chi}) L(3, \bar{\chi})=\frac{\phi(q)}{2^{3 i+1}} D_{k}(2, i) \prod_{p \nmid 2 q} D_{k}(p, 0)+O\left(q^{\varepsilon}\right) .
$$

Proof. We only prove the second formula, since the first one can be deduced similarly. Let $d_{k}(n)$ be the $k$ th divisor function (i.e., the number of positive integer solutions of the equation $\left.n_{1} \cdots n_{k}=n\right)$, and define $\tau_{k}(n)=$ $\sum_{d \mid n} d^{2} d_{k}(d)$. For any nonprincipal character $\chi$ modulo $q$, and parameter $N \geq q$, applying Abel's identity we have

$$
\begin{aligned}
L(s, \chi) & =\sum_{n=1}^{\infty} \frac{\chi(n)}{n^{s}}=\sum_{1 \leq n \leq N} \frac{\chi(n)}{n^{s}}+s \int_{N}^{\infty} \frac{\sum_{N<n \leq y} \chi(n)}{y^{s+1}} d y \\
& =\sum_{1 \leq n \leq N} \frac{\chi(n)}{n^{s}}+O\left(\frac{\sqrt{q} \log q}{N^{s}}\right) .
\end{aligned}
$$

Noting that for $(a b, q)=1$, by the orthogonality relations for character sums modulo $q$ we get

$$
\sum_{\substack{\chi \bmod q \\ \chi(-1)=-1}} \chi(a) \bar{\chi}(b)= \begin{cases}\frac{1}{2} \phi(q) & \text { if } a \equiv b \bmod q \\ -\frac{1}{2} \phi(q) & \text { if } a \equiv-b \bmod q \\ 0 & \text { otherwise. }\end{cases}
$$

Then

$$
\begin{aligned}
\Sigma & :=\sum_{\substack{\chi \bmod q \\
\chi(-1)=-1}} \chi\left(2^{i}\right) L^{k+1}(1, \chi) L^{k}(1, \bar{\chi}) L(3, \bar{\chi}) \\
= & \sum_{\substack{\chi \bmod q \\
\chi(-1)=-1}} \chi\left(2^{i}\right)\left[\sum_{1 \leq n \leq N} \frac{\chi(n)}{n}+O\left(\frac{\sqrt{q} \log q}{N}\right)\right]^{k+1} \\
& \times\left[\sum_{1 \leq m_{1} \leq N} \frac{\bar{\chi}\left(m_{1}\right)}{m_{1}}+O\left(\frac{\sqrt{q} \log q}{N}\right)\right]^{k}\left[\sum_{1 \leq m_{2} \leq N} \frac{\bar{\chi}\left(m_{2}\right)}{m_{2}^{3}}+O\left(\frac{\sqrt{q} \log q}{N^{3}}\right)\right]
\end{aligned}
$$




$$
\begin{aligned}
& =\sum_{\substack{\chi \bmod q \\
\chi(-1)=-1}} \chi\left(2^{i}\right)\left(\sum_{1 \leq n \leq N} \frac{\chi(n)}{n}\right)^{k+1}\left(\sum_{1 \leq m_{1} \leq N} \frac{\bar{\chi}\left(m_{1}\right)}{m_{1}}\right)^{k}\left(\sum_{1 \leq m_{2} \leq N} \frac{\bar{\chi}\left(m_{2}\right)}{m_{2}^{3}}\right) \\
& +O\left(\frac{q^{3 / 2} \log ^{2 k} N \log q}{N}\right) \\
& =\sum_{\substack{\chi \bmod q \\
\chi(-1)=-1}} \chi\left(2^{i}\right) \sum_{1 \leq n \leq N^{k+1}} \frac{\chi(n) d_{k+1}(n)}{n} \sum_{1 \leq m \leq N^{k+1}} \frac{\bar{\chi}(m)}{m} \sum_{d \mid m} \frac{d_{k}(m / d)}{d^{2}} \\
& +O\left(\frac{q^{3 / 2} \log ^{2 k} N \log q}{N}\right) \\
& =\frac{\phi(q)}{2} \sum_{\substack{1 \leq n \leq N^{k+1} \\
(n, q)=1 \\
m \equiv 2^{i} n \bmod q}} \sum_{\substack{1 \leq m \leq N^{k+1} \\
(m, q)=1}} \frac{d_{k+1}(n)}{n} \frac{\sum_{d \mid m} d_{k}(m / d) / d^{2}}{m} \\
& -\frac{\phi(q)}{2} \sum_{\substack{1 \leq n \leq N^{k+1} \\
(n, q)=1 \\
m \equiv-2^{i} n \cos q}} \sum_{\substack{1 \leq m \leq N^{k+1} \\
(m, q)=1}} \frac{d_{k+1}(n)}{n} \frac{\sum_{d \mid m} d_{k}(m / d) / d^{2}}{m} \\
& +O\left(\frac{q^{3 / 2} \log ^{2 k} N \log q}{N}\right) \\
& =\frac{\phi(q)}{2} \sum_{\substack{1 \leq n \leq N^{k+1} \\
(n, q)=1}} \frac{d_{k+1}(n)}{2^{i} n^{2}} \sum_{d \mid 2^{i} n} \frac{d_{k}\left(2^{i} n / d\right)}{d^{2}}+O\left(\frac{q^{3 / 2} \log ^{2 k} N \log q}{N}\right) \\
& +O\left(\phi(q) \sum_{1 \leq n \leq N^{k+1}} \frac{d_{k+1}(n)}{n} \Sigma_{1}\right) \\
& +O\left(\phi(q) \sum_{1 \leq n \leq N^{k+1}} \frac{d_{k+1}(n)}{n} \Sigma_{2}\right)
\end{aligned}
$$

where

$$
\begin{aligned}
\Sigma_{1} & =\sum_{\frac{1-2^{i} n}{q} \leq l \leq{\frac{N}{k+1}-2^{i} n}_{l \neq 0}^{q}} \frac{\sum_{d \mid l q+2^{i} n} d_{k}\left(\left(l q+2^{i} n\right) / d\right) / d^{2}}{l q+2^{i} n}, \\
\Sigma_{2} & =\sum_{\frac{1+2^{i} n}{q} \leq l \leq \frac{N^{k+1}+2^{i} n}{q}} \frac{\sum_{d \mid l q-2^{i} n} d_{k}\left(\left(l q-2^{i} n\right) / d\right) / d^{2}}{l q-2^{i} n} .
\end{aligned}
$$


Hence

$$
\Sigma=\frac{\phi(q)}{2^{3 i+1}} \sum_{\substack{n=1 \\(n, q)=1}}^{\infty} \frac{d_{k+1}(n) \tau_{k}\left(2^{i} n\right)}{n^{4}}+O\left(\frac{q^{3 / 2} \log ^{2 k} N \log q}{N}\right)+O\left(N^{\varepsilon}\right) .
$$

Now taking $N=q^{3 / 2}$, we immediately get

$$
\Sigma=\frac{\phi(q)}{2^{3 i+1}} \sum_{\substack{n=1 \\(n, q)=1}}^{\infty} \frac{d_{k+1}(n) \tau_{k}\left(2^{i} n\right)}{n^{4}}+O\left(q^{\varepsilon}\right) .
$$

Noting that $d_{k}\left(p^{r}\right)=C_{k+r-1}^{k-1}($ see $[4,6.4 .12])$, from the properties of multiplicative functions we have

$$
\text { 17) } \begin{aligned}
& \sum_{\substack{n=1 \\
(n, q)=1}}^{\infty} \frac{d_{k+1}(n) \tau_{k}\left(2^{i} n\right)}{n^{4}}=\sum_{\substack{n=1 \\
(n, 2 q)=1}}^{\infty} \frac{d_{k+1}(n) \tau_{k}\left(2^{i} n\right)}{n^{4}} \\
+ & \sum_{\substack{n=1 \\
(n, 2 q)=1}}^{\infty} \frac{d_{k+1}(2 n) \tau_{k}\left(2^{i+1} n\right)}{(2 n)^{4}}+\cdots+\sum_{\substack{n=1 \\
(n, 2 q)=1}}^{\infty} \frac{d_{k+1}\left(2^{j} n\right) \tau_{k}\left(2^{i+j} n\right)}{\left(2^{j} n\right)^{4}}+\cdots \\
= & {\left[\sum_{\substack{n=1 \\
(n, 2 q)=1}}^{\infty} \frac{d_{k+1}(n) \tau_{k}(n)}{n^{4}}\right] } \\
\times & {\left[\tau_{k}\left(2^{i}\right)+\frac{d_{k+1}(2) \tau_{k}\left(2^{i+1}\right)}{2^{4}}+\cdots+\frac{d_{k+1}\left(2^{m}\right) \tau_{k}\left(2^{i+m}\right)}{2^{4 m}}+\cdots\right] }
\end{aligned}
$$

$=: S_{1} \cdot S_{2}$.

By applying Euler products we get

$$
\begin{aligned}
S_{1} & =\prod_{p \nmid 2 q}\left[1+\frac{d_{k+1}(p) \tau_{k}(p)}{p^{4}}+\cdots+\frac{d_{k+1}\left(p^{n}\right) \tau_{k}\left(p^{n}\right)}{p^{4 n}}+\cdots\right] \\
& =\prod_{p \nmid 2 q}\left[1+\cdots+\frac{C_{k+n}^{k} \sum_{j=0}^{n} p^{2 j} C_{k+j-1}^{k-1}}{p^{4 n}}+\cdots\right] \\
& =\prod_{p \nmid 2 q}\left[\sum_{n=0}^{\infty} \frac{C_{k+n}^{k}}{p^{4 n}} \sum_{j=0}^{n} p^{2 j} C_{k+j-1}^{k-1}\right] \\
& =\prod_{p \nmid 2 q} D_{k}(p, 0) .
\end{aligned}
$$


On the other hand, it is not hard to show that

$$
\begin{aligned}
S_{2}= & \tau_{k}\left(2^{i}\right)+\frac{d_{k+1}(2) \tau_{k}\left(2^{i+1}\right)}{2^{4}}+\cdots+\frac{d_{k+1}\left(2^{m}\right) \tau_{k}\left(2^{i+m}\right)}{2^{4 m}}+\cdots \\
= & \sum_{j=0}^{i} 2^{2 j} C_{k+j-1}^{k-1}+\frac{C_{k+1}^{k} \sum_{j=0}^{i+1} 2^{2 j} C_{k+j-1}^{k-1}}{2^{4}} \\
& +\cdots+\frac{C_{k+m}^{k} \sum_{j=0}^{i+m} 2^{2 j} C_{k+j-1}^{k-1}}{2^{4 m}}+\cdots \\
= & \sum_{n=0}^{\infty} \frac{C_{k+n}^{k}}{2^{4 n}} \sum_{j=0}^{i+n} 2^{2 j} C_{k+j-1}^{k-1}=D_{k}(2, i) .
\end{aligned}
$$

Then from (16)-(19) we get the second formula of Lemma 5.

Lemma 6. Let $p$ be an odd prime. Then

$$
\begin{aligned}
& \sum_{\substack{\chi \bmod p \\
\chi(-1)=-1}}|L(1, \bar{\chi})|^{4}=\frac{5 \pi^{4}}{144} p+O\left(p^{\varepsilon}\right), \\
& \sum_{\substack{\chi \bmod p \\
\chi(-1)=-1}} \chi(2)|L(1, \bar{\chi})|^{4}=\frac{\pi^{4}}{36} p+O\left(p^{\varepsilon}\right), \\
& \sum_{\substack{\chi \bmod p \\
\chi(-1)=-1}} \chi(4)|L(1, \bar{\chi})|^{4}=\frac{11 \pi^{4}}{576} p+O\left(p^{\varepsilon}\right), \\
& \sum_{\substack{\chi \bmod p \\
\chi(-1)=-1}} L^{2}(1, \chi) L(1, \bar{\chi}) L(3, \bar{\chi})=\frac{7 \pi^{6}}{4320} p+O\left(p^{\varepsilon}\right), \\
& \sum_{\substack{\chi \bmod p \\
\chi(-1)=-1}} \chi(2) L^{2}(1, \chi) L(1, \bar{\chi}) L(3, \bar{\chi})=\frac{\pi^{6}}{1080} p+O\left(p^{\varepsilon}\right), \\
& \sum_{\substack{\chi \bmod p \\
\chi(-1)=-1}} \chi(4) L^{2}(1, \chi) L(1, \bar{\chi}) L(3, \bar{\chi})=\frac{11 \pi^{6}}{23040} p+O\left(p^{\varepsilon}\right) . \\
& \sum_{\substack{\chi \bmod p \\
\chi(-1)=-1}} \chi\left(2^{i}\right)|L(1, \bar{\chi})|^{4}=C_{1}(i) \zeta^{3}(2) p \prod_{p_{1} \nmid 2 p}\left(1-\frac{1-C_{2}^{1}}{p_{1}^{2}}\right)+O\left(p^{\varepsilon}\right) \\
& =C_{1}(i) \zeta^{3}(2) p \prod_{p_{1} \nmid 2 p}\left(1+\frac{1}{p_{1}^{2}}\right)+O\left(p^{\varepsilon}\right)
\end{aligned}
$$




$$
\begin{aligned}
& =\frac{4}{5} \zeta^{3}(2) C_{1}(i) p \prod_{p_{1}}\left(1+\frac{1}{p_{1}^{2}}\right)+O\left(p^{\varepsilon}\right) \\
& =\frac{4}{5} \frac{\zeta^{4}(2)}{\zeta(4)} C_{1}(i) p+O\left(p^{\varepsilon}\right)=\frac{1}{18} \pi^{4} C_{1}(i) p+O\left(p^{\varepsilon}\right) .
\end{aligned}
$$

Noting that

$$
\begin{aligned}
C_{1}(i) & =\frac{1}{2^{i+1}}\left[\left(1-\frac{1}{2^{2}}\right) C_{1+i}^{1}+\frac{C_{2}^{1}}{2^{2}}\right] \\
& =\frac{1}{2^{i+1}}\left[\frac{3(1+i)}{4}+\frac{2}{4}\right]=\frac{5+3 i}{2^{i+3}},
\end{aligned}
$$

we obtain the first three formulas of the conclusion.

On the other hand, from the definition of $D_{k}(p, i)$ we have

$$
\begin{aligned}
D_{1}(p, i) & =\sum_{n=0}^{\infty} \frac{C_{1+n}^{1}}{p^{4 n}} \sum_{j=0}^{i+n} p^{2 j} \\
& =\sum_{n=0}^{\infty} \frac{n+1}{p^{4 n}} \frac{p^{2(n+i+1)}-1}{p^{2}-1} \\
& =\frac{1}{p^{2}-1}\left[p^{2 i+2} \sum_{n=0}^{\infty} \frac{n+1}{p^{2 n}}-\sum_{n=0}^{\infty} \frac{n+1}{p^{4 n}}\right] \\
& =\frac{1}{p^{2}-1}\left[p^{2 i+2} \frac{1}{\left(1-1 / p^{2}\right)^{2}}-\frac{1}{\left(1-1 / p^{4}\right)^{2}}\right] .
\end{aligned}
$$

Therefore

$$
\begin{aligned}
D_{1}\left(p_{1}, 0\right) & =\frac{1}{p_{1}^{2}-1}\left[p_{1}^{2} \frac{1}{\left(1-1 / p_{1}^{2}\right)^{2}}-\frac{1}{\left(1-1 / p_{1}^{4}\right)^{2}}\right] \\
& =\frac{1}{\left(1-1 / p_{1}^{2}\right)^{3}}\left[1-\frac{1}{p_{1}^{2}\left(1+1 / p_{1}^{2}\right)^{2}}\right] \\
& =\frac{1-1 / p_{1}^{6}}{\left(1-1 / p_{1}^{2}\right)^{4}\left(1+1 / p_{1}^{2}\right)^{2}}
\end{aligned}
$$

and

$$
\begin{aligned}
D_{1}(2, i) & =\frac{1}{2^{2}-1}\left[2^{2 i+2} \frac{1}{\left(1-1 / 2^{2}\right)^{2}}-\frac{1}{\left(1-1 / 2^{4}\right)^{2}}\right] \\
& =\frac{2^{2 i} \cdot 1600-256}{675} .
\end{aligned}
$$


Then we get

$$
\begin{aligned}
\sum_{\substack{\chi \bmod p \\
\chi(-1)=-1}} \chi\left(2^{i}\right) L^{2}(1, \chi) L(1, \bar{\chi}) L(3, \bar{\chi})=\frac{p}{2^{3 i+1}} D_{1}(2, i) \prod_{p_{1} \nmid 2 p} D_{1}\left(p_{1}, 0\right)+O\left(p^{\varepsilon}\right) \\
\quad=\frac{2^{2 i} \cdot 1600-256}{675 \cdot 2^{3 i+1}} p \prod_{p_{1} \nmid 2 p} \frac{1-1 / p_{1}^{6}}{\left(1-1 / p_{1}^{2}\right)^{4}\left(1+1 / p_{1}^{2}\right)^{2}}+O\left(p^{\varepsilon}\right) \\
\quad=\frac{2^{2 i} \cdot 25-4}{2^{3 i} \cdot 42} p \prod_{p_{1}} \frac{1-1 / p_{1}^{6}}{\left(1-1 / p_{1}^{2}\right)^{4}\left(1+1 / p_{1}^{2}\right)^{2}}+O\left(p^{\varepsilon}\right) \\
=\frac{2^{2 i} \cdot 25-4}{2^{3 i} \cdot 42} \frac{\zeta^{2}(2) \zeta^{2}(4)}{\zeta(6)} p+O\left(p^{\varepsilon}\right)=\frac{\left(2^{2 i} \cdot 25-4\right) \pi^{6}}{2^{3 i} \cdot 6 \cdot 2160} p+O\left(p^{\varepsilon}\right) .
\end{aligned}
$$

This yields the last three formulas of Lemma 6 .

3. Proof of the theorems. In this section, we complete the proof of the theorems. For any prime $p \geq 3$, note that

$$
\tau(\chi) \tau(\bar{\chi})=p \chi(-1) .
$$

Then from Lemmas 1, 2, 4, 6 and the orthogonality relation for character sums modulo $p$ we get

$$
\begin{aligned}
& \sum_{c=1}^{p-1} E(p, 1, c) C(c, 1, p) \\
&=\frac{1}{\pi^{2}(p-1)} \sum_{\chi(-1)=-1} \tau^{2}(\bar{\chi}) L^{2}(1, \chi)\left[\left(\sum_{a=1}^{p} a \chi(a)\right)^{2}\right. \\
&\left.+\left(\sum_{a=1}^{p}(-1)^{a} a^{2} \chi(a)\right)\left(\sum_{b=1}^{p}(-1)^{b} \chi(b)\right)-\left(\sum_{a=1}^{p}(-1)^{a} a \chi(a)\right)^{2}\right] \\
&+O\left(p^{5 / 2+\varepsilon}\right) \\
&=\frac{p^{4}}{\pi^{4}(p-1)} \sum_{(-1)=-1}(-2+4 \chi(2)-4 \chi(4))|L(1, \bar{\chi})|^{4} \\
&+\frac{p^{4}}{\pi^{6}(p-1)} \sum_{\chi(-1)=-1}(2-20 \chi(2)+32 \chi(4)) L^{2}(1, \chi) L(1, \bar{\chi}) L(3, \bar{\chi}) \\
&+ O\left(p^{7 / 2+\varepsilon}\right) \\
&=-\frac{5}{144} p^{4}+O\left(p^{7 / 2+\varepsilon}\right) .
\end{aligned}
$$

This proves Theorem 1 . 
For any prime $p \geq 3$ and integer $k \geq 2$, from Lemmas 1 and $3-5$ we have

$$
\begin{aligned}
& \sum_{c=1}^{p-1} E(p, k, c) C(c, k, p)=-\frac{i^{k+1}}{2 \pi^{k+1}(p-1)} \sum_{\chi(-1)=-1} \tau^{k+1}(\bar{\chi}) L^{k+1}(1, \chi) \\
& \times\left[(k+1)\left(\sum_{a=1}^{p}(-1)^{a} \chi(a)\right)^{k}\left(\sum_{b=1}^{p}(-1)^{b} b^{2} \chi(b)\right)\right. \\
& \left.+k(k-3)\left(\sum_{a=1}^{p}(-1)^{a} a \chi(a)\right)^{2}\left(\sum_{b=1}^{p}(-1)^{b} \chi(b)\right)^{k-1}\right] \\
& =-\frac{2^{k-2}\left(k^{2}-k+2\right) p^{k+3}}{\pi^{2 k+2}(p-1)} \\
& \times \sum_{\chi(-1)=-1}(1-2 \chi(2))^{k+1}|L(1, \bar{\chi})|^{2 k+2}+O\left(p^{k+5 / 2+\varepsilon}\right) \\
& +\frac{2^{k-1}(k+1) p^{k+3}}{\pi^{2 k+4}(p-1)} \\
& \times \sum_{\chi(-1)=-1}(1-2 \chi(2))^{k}(1-8 \chi(2)) L^{k+1}(1, \chi) L^{k}(1, \bar{\chi}) L(3, \bar{\chi}) \\
& =-\frac{2^{k-2}\left(k^{2}-k+2\right) p^{k+3}}{\pi^{2 k+2}(p-1)} \sum_{i=0}^{k+1} C_{k+1}^{i}(-2)^{i} \sum_{\chi(-1)=-1} \chi\left(2^{i}\right)|L(1, \bar{\chi})|^{2 k+2} \\
& +O\left(p^{k+5 / 2+\varepsilon}\right) \\
& +\frac{2^{k-1}(k+1) p^{k+3}}{\pi^{2 k+4}(p-1)} \sum_{\chi(-1)=-1}\left[\sum_{i=0}^{k} C_{k}^{i}(-2)^{i} \chi\left(2^{i}\right)+\sum_{j=0}^{k} C_{k}^{j}(-2)^{j+3} \chi\left(2^{j+1}\right)\right] \\
& \times L^{k+1}(1, \chi) L^{k}(1, \bar{\chi}) L(3, \bar{\chi}) \\
& =-\frac{2^{k-2}\left(k^{2}-k+2\right) \zeta^{2 k+1}(2) p^{k+3}}{\pi^{2 k+2}}\left[\sum_{i=0}^{k+1} C_{k+1}^{i}(-2)^{i} C_{k}(i)\right] \prod_{p_{1} \nmid 2 p}\left(1-\frac{1-C_{2 k}^{k}}{p_{1}^{2}}\right) \\
& +\frac{2^{k-1}(k+1) p^{k+3}}{\pi^{2 k+4}} \\
& \times\left[\sum_{i=0}^{k} C_{k}^{i}(-2)^{i} \frac{D_{k}(2, i)}{2^{3 i+1}}+\sum_{j=0}^{k} C_{k}^{j}(-2)^{j+3} \frac{D_{k}(2, j+1)}{2^{3 j+4}}\right] \prod_{p_{1} \nmid 2 p} D_{k}\left(p_{1}, 0\right) \\
& +O\left(p^{k+5 / 2+\varepsilon}\right) \text {. }
\end{aligned}
$$

This completes the proof of Theorem 2 . 
Acknowledgments. The authors express their gratitude to the referee for his very helpful and detailed comments on improving this paper.

\section{References}

[1] T. M. Apostol, Introduction to Analytic Number Theory, Springer, New York, 1976.

[2] T. Funakura, On Kronecker's limit formula for Dirichlet series with periodic coefficients, Acta Arith. 55 (1990), 59-73.

[3] R. K. Guy, Unsolved Problems in Number Theory, Springer, New York, 1994.

[4] Chengdong Pan and Chengbiao Pan, Elements of the Analytic Number Theory, Science Press, Beijing, 1991.

[5] G. Pólya, Über die Verteilung der quadratischen Reste und Nichtreste, Göttinger Nachr. 1918, 21-29.

[6] W. P. Zhang, A problem of D. H. Lehmer and its generalization (I), Compos. Math. 86 (1993), 307-316.

[7] -, A problem of D. H. Lehmer and its generalization (II), ibid. 91 (1994), 47-56.

[8] - On the difference between a D. H. Lehmer number and its inverse modulo q, Acta Arith. 68 (1994), 255-263.

[9] —, On a Cochrane sum and its hybrid mean value formula, J. Math. Anal. Appl. 267 (2002), 89-96.

[10] —, On a Cochrane sum and its hybrid mean value formula (II), ibid. 276 (2002), 446-457.

[11] -, A problem of D. H. Lehmer and its mean square value formula, Japan. J. Math. 29 (2003), 109-116.

[12] -, On a problem of D. H. Lehmer and Kloosterman sums, Monatsh. Math. 139 (2003), 247-257.

[13] - A sum analogous to Dedekind sums and its hybrid mean value formula, Acta Arith. 107 (2003), 1-8.

[14] W. P. Zhang and Y. Yi, On the upper bound estimate of Cochrane sums, Soochow J. Math. 28 (2002), 297-304.

Department of Mathematics

Northwest University

Xi'an, Shaanxi, P.R. China

E-mail: hnliumath@hotmail.com

wpzhang@nwu.edu.cn

Received on 16.12.2003

and in revised form on 15.9.2006 